(C) 2016 IEEE. Personal use of this material is permitted. Permission from IEEE must be obtained for all other uses, in any current or future media, including reprinting/republishing this material for advertising or promotional purposes, creating new collective works, for resale or redistribution to servers or lists, or reuse of any copyrighted component of this work in other works. 


\title{
A Model Predictive Power Control Method with Longer Prediction Horizon for Distributed Power Generations
}

\author{
Mahlagha Mahdavi Aghdam, Student Member, IEEE, Li Li, Member, IEEE, Jianguo Zhu, Senior Member, IEEE \\ Centre of Green Energy and Vehicle Innovation, University of Technology Sydney, Ultimo, NSW 2007, Australia \\ Mahlagha.MahdaviAghdam@student.uts.edu.au
}

\begin{abstract}
The model predictive control method can be effective for converter control in distributed power generation but requires a large amount of computation, leading to a considerable time delay in the actuation. If the delay is not reflected, the system performance could get worse. This paper presents a two-step (horizon) prediction algorithm of model predictive control technique for grid-tied inverters used in wind turbine systems. The control objectives such as active and reactive power flow and switching loss reduction are reflected in the objective function of the controller. The proposed model predictive control strategy is verified numerically by using MATLAB/Simulink.
\end{abstract}

Keywords-Distributed Generation; Grid-Tied Power Inverter; Model Predictive Control; Prediction Horizon;

\section{INTRODUCTION}

The increasing number of distributed generations (DGs) in the neighborhood of consumers, which is also known as prosumer, imposes new challenges for the operation and management of these systems as well as the utility grid to sustain or enhance the power quality and reliability. The power electronic technology has a critical feature in various industrial applications such as integration of renewable energy sources (RESs) into the power system. Power electronics have gone through a fast development, generally due to advances in power-semiconductor switches along with high performance control algorithms [1].

Control approaches for power converters have been the focus of continuing investigation for about several decades in power electronics topics. Some control schemes have been established and implemented on numerous power converter topologies for low, medium and high power applications such as non-linear controller based on hysteresis bounds and linear control with modulators. Although these methodologies bring some benefits, complex coordinate transformation is required and much regulation effort is an essential to guarantee the system stability [2]. However, with the advancement of faster, more accurate and more powerful microprocessors, using digital control platforms can unlock new horizons for power converter control. Some of these algorithms which have been applied to power converters are artificial intelligence-based control [3], sliding mode control [4, 5], and predictive control [6].

In modern industrial controls, model predictive control (MPC) scheme has been implemented in hundreds of realworld applications [7, 8]. MPC techniques applied to power converters can be considered in two categories: (a)the finite control set MPC approach, which takes the benefit of the limited number of switching states of the power converter to optimize the problem, and (b) the continuous control set MPC approach, which needs a modulator to produce the switching states from the continuous output of the controller [9]. The finite control set MPC has become an intuitive and powerful algorithm to control power converters, without the need for linear PI regulators and modulators. In this paper, we assume the MPC is based on finite control set method.

Generally, MPC calculates, at each sampling instant, an optimal control problem over a finite prediction window. The optimization of the objectives leads to an optimal control sequence for the whole prediction horizon. At each sampling instant, this process is repeated with new state estimations or measurements [10]. Although, MPC is able to handle the system nonlinearities and constraints in the same time, a mathematical model of the system, either single input-single output or multiple inputs-multiple outputs, is an essential to implement the MPC.

A noteworthy weakness of MPC is that developing the discretely manipulated variable demonstrates to be a computational challenge. The computational concerns become predominantly significant for longer prediction horizons, since the number of possible switching sequences rises exponentially as the horizon length is increased.

This paper presents a two-step MPC method for gridconnected power converters, which can provide a fast optimization for longer prediction horizons. The theory and principle of the proposed MPC are presented in Section II. A two-level three-phase voltage source inverter topology is chosen as the benchmark to perform the MPC in Section III. Section IV applies the MPC to a case study by using the direct power control and considering the time delay. A quantitative comparison of MPC with and without time compensation is conducted in Section V. Finally, Section VI is devoted to the conclusion and the future work. 


\section{THEORY OF MPC FOR POWER CONVERTERS}

\section{A. MPC Algorithm}

As mentioned before, MPC is a model based control technique; thus, developing an acceptable model of the plant is a key. As power electronics applications are commonly controlled by using digital platforms, it is fitting to model the system in discrete-time domain state space form. Therefore, the mathematical analysis of the system is narrowed to the discrete-time domain. For an r-level s-phase converter, the total number of possible switching states is $m=r^{s}$. The dynamics of the power converter system in the discrete time domain can be written as

$$
\begin{gathered}
\boldsymbol{x}_{\boldsymbol{i}}(k+1)=f\left(\boldsymbol{x}_{\boldsymbol{i}}(k), \boldsymbol{u}_{\boldsymbol{i}}(k)\right) \\
\boldsymbol{y}_{\boldsymbol{i}}(k)=g\left(\boldsymbol{x}_{\boldsymbol{i}}(k)\right)
\end{gathered} \quad \text { for } i=1, \ldots, m .
$$

where $\boldsymbol{x}_{\boldsymbol{i}}(k), \boldsymbol{u}_{\boldsymbol{i}}(k)$, and $\boldsymbol{y}_{\boldsymbol{i}}(k)$ are the state, input, and output vector variables at the time instant $k T_{s}$, respectively, and $T_{s}$ is the sampling interval. The state variables are available through measurement and estimation, which provides the current plant information, at the sampling instant $k(k>0)$. In one sampling time ahead, the predicted state $\boldsymbol{x}_{\boldsymbol{i}}(k+1)$ can be obtained based on a prediction function $f$. The future state variables at the step $k+N$, where $N$ is the prediction horizon, can be represented as

$$
\begin{gathered}
\boldsymbol{x}_{\boldsymbol{i}}(k+N)=f\left(\boldsymbol{x}_{\boldsymbol{i}}(k+N-1), \boldsymbol{u}_{\boldsymbol{i}}(k+N-1)\right) \\
\boldsymbol{y}_{\boldsymbol{i}}(k+N)=g\left(\boldsymbol{x}_{\boldsymbol{i}}(k+N)\right)
\end{gathered}
$$

Apart from predicting the future state within the finite horizon, incorporating constraints in the controller can improve the system performance effectively [11]. In this way the plant is able to operate at its physical limits without violating them. Thus, the most appropriate operation can be gained, while the operational constrains of the plant are enforced. Constraints can be imposed to the state variables and/or to the input variables.

The control objective should be clearly determined. In the case of tracking the reference, $\boldsymbol{x}_{i}^{*}(k+1)$, over a finite prediction horizon $N$, the control problem can be realized via minimization of the objective function

$$
\begin{aligned}
J_{i}= & \sum_{l=k}^{k+N-1}\left\|\boldsymbol{x}_{i, E r r}(l+1)\right\|_{2}^{2} \quad \text { for } i=1, \ldots, m . \\
& \boldsymbol{x}_{i, E r r}(l+1) \triangleq \boldsymbol{x}_{i}^{*}(l+1)-x_{i}(l+1)
\end{aligned}
$$

The valuation of the cost function with the $m$ predictions will lead to $m$ different costs. Certainly, the control action will be chosen as the one which leads to the minimum cost ( $\mathrm{min}$ $\left\{J_{i}\right\}$, for $\left.i=1, \ldots, m\right)$.

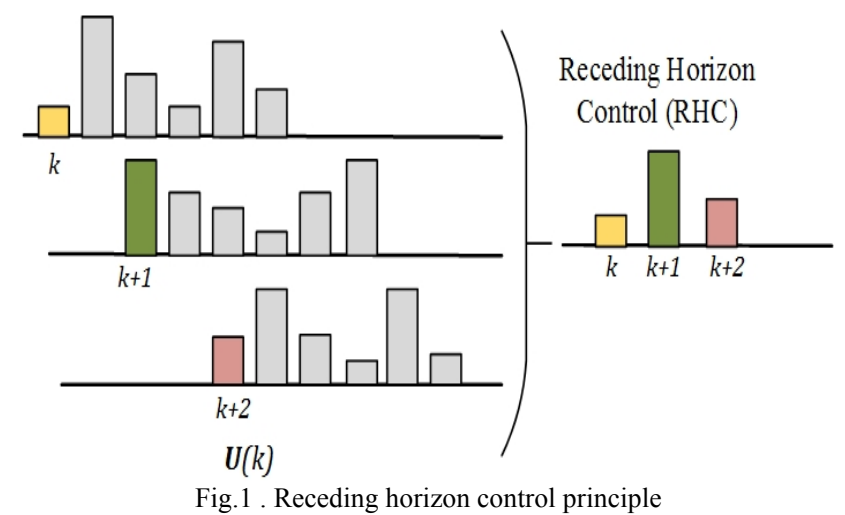

The switching sequence can be defined as

$$
\boldsymbol{U}(k)=\left[u^{T}(k) \ldots u^{T}(k+N-1)\right]^{T}
$$

which characterizes the sequence of power converter switch positions. The optimization problem can then be stated as

$$
\boldsymbol{U}_{\text {opt }}(k)=\underset{\boldsymbol{U}(k)}{\arg \min } J_{i}
$$

The cost function $J$ depends on the state vector $\boldsymbol{x}(k)$, the last chosen switch position $u(k-1)$ and the switching sequence $\boldsymbol{U}(k)$. Subsequent to the receding horizon optimization principle, as can be seen in Fig.1, the first element of the optimizing sequence $\boldsymbol{U}_{\text {opt }}(k)$ is applied to the semiconductor switches at time step $k$.

MPC is a flexible control scheme that permits the easy presence of system objectives; this has raised much interest in recent years [12]. For example, in MPC, switching losses can be controlled by adding another term to the cost function $J_{i}$.

\section{B. Model Predictive Control Issues}

In term of the real-time implementation, the time needed to calculate the control signal may take a significant share of the sampling time, causing one sampling time delay. The time delay has a huge influence on the prediction, particularly when a one-step horizon algorithm is in place. Therefore, a delay compensation pattern must be included in the controller.

In terms of the length of prediction horizon, there is no theoretical boundary to the number of predictions that can be executed. Though, practical implementations are restricted by the computational requirements, which will impose a maximum number of reachable predictions [13].

In terms of the weighting factors for multi-objective functions, the method of finding the weighting factor is only empirical. Another approach for compensating the unit difference is normalizing each component in order to eliminate their unit effects.

\section{CASE Study of A Grid-Tied InVERTER System}

A wind turbine (WT) system connected to the grid through an inverter is shown in Fig.2. In general, the WT system 
consists of an $\mathrm{AC} / \mathrm{DC}$ converter and an inverter. The maximum power tracking is performed by the $\mathrm{AC} / \mathrm{DC}$ converter whereas the inverter is used to convert the $\mathrm{DC}$ voltage to AC voltage.

Whilst the concept, general arrangement and operating principle of the present work can be applied to $\mathrm{AC} / \mathrm{DC}$, and $\mathrm{DC} / \mathrm{AC}$ topologies, including active front ends, neutral point clamped converters, multi-level converters, inverters with AC machines and drives, we will focus on the arrangement described below.

\section{A. Physical Model of the Grid-Connected Inverter}

One of the most common DC/AC converter topologies used in distributed power generations is the two-level threephase voltage source inverter (2L-3Ph VSI). The topology of the $2 \mathrm{~L}-3 \mathrm{Ph}$ grid-tied inverter is depicted in Fig. 3. It is formed by three complementary pairs of IGBT power switches, and is connected to the utility grid via an RL filter and also a circuit breaker for the protection purpose. Thus, in this case, eight possible switching states can be found. The output voltage space vectors generated by the inverter are defined by

$$
v_{m}=\left\{\begin{aligned}
\frac{2}{3} e^{j(m-1) \frac{\pi}{3}} V_{d c}, & & m=1, \ldots, 6 \\
0, & m & =0,7
\end{aligned}\right.
$$

The voltage vectors generated by the inverter are comprised of six active $(V 1-V 6)$ and two zero $(V 0, V 7)$ voltage vectors, as illustrated in Fig. 4.

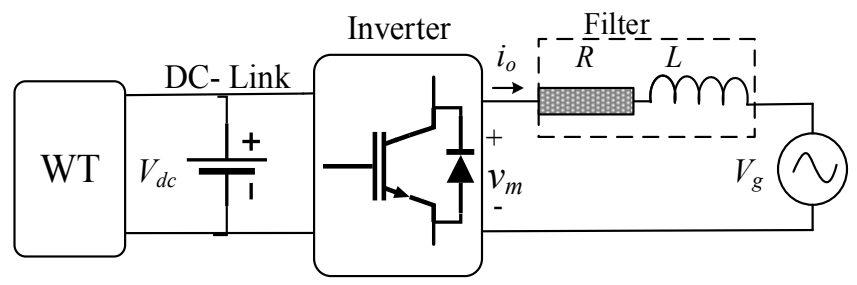

Fig. 2. One-phase model of the grid-Tied wind turbine system

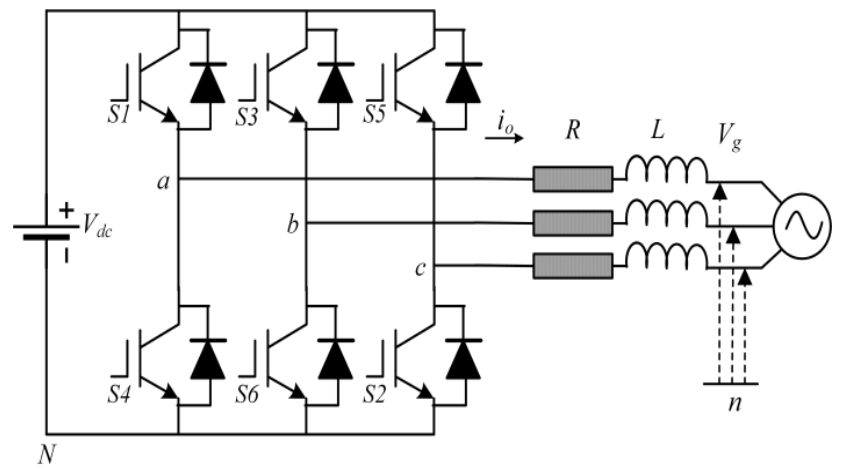

Fig. 3. Two-level three-phase grid-tied inverter

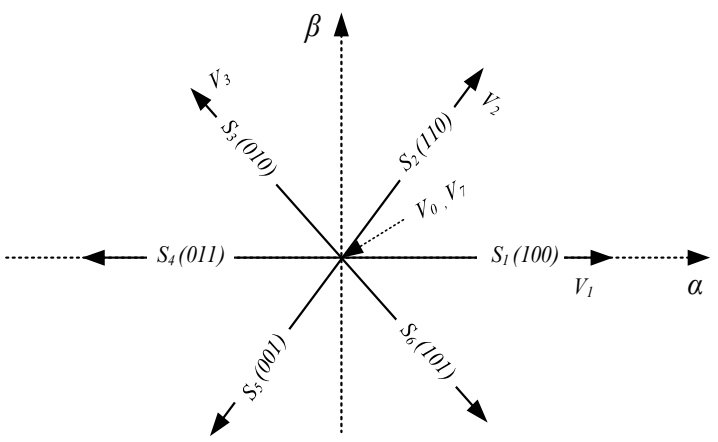

Fig. 4. Voltage vectors generated by the inverter

\section{B. Mathematical Model} is

The mathematical equation of the grid-connected inverter

$$
V_{m}=L \frac{d i_{o}}{d t}+R \cdot i_{0}+V_{g}
$$

where $V_{m}, i_{o}$ and $V_{g}$ represent the inverter terminal voltage, phase current and grid voltage, respectively. By applying the Clarke transformation all variables $\xi_{a b c}=\left[\begin{array}{lll}\xi_{a} & \xi_{b} & \xi_{c}\end{array}\right]^{T}$ in three phase system are converted into $\xi_{\alpha \beta}=\left[\begin{array}{ll}\xi_{\alpha} & \xi_{\beta}\end{array}\right]^{T}$, a stationary two-phase reference frame as,

$$
\xi_{\alpha \beta}=\left(\begin{array}{ccc}
1 & -\frac{1}{2} & -\frac{1}{2} \\
0 & \frac{\sqrt{3}}{2} & -\frac{\sqrt{3}}{2}
\end{array}\right) \xi_{a b c}
$$

The state-space model of the system in continuous-time domain can be written as

$$
\frac{d x}{d t}=D x+F u
$$

where,

$$
x=\left[\begin{array}{llll}
i_{o \alpha} & i_{o \beta} & V_{g \alpha} & V_{g \beta}
\end{array}\right]^{T}, \quad u=\left[\begin{array}{ll}
V_{m \alpha} & V_{m \beta}
\end{array}\right]^{T}
$$

and

$$
D=\left(\begin{array}{cccc}
-\frac{R}{L} & 0 & -\frac{1}{L} & 0 \\
0 & -\frac{R}{L} & 0 & -\frac{1}{L} \\
0 & 0 & 0 & -\omega \\
0 & 0 & \omega & 0
\end{array}\right), F=\left(\begin{array}{cc}
\frac{1}{L} & 0 \\
0 & \frac{1}{L} \\
0 & 0 \\
0 & 0
\end{array}\right)
$$

The discrete time representation of the system is,

$$
x(k+1)=A x(k)+B u(k)
$$

where $A \triangleq I+D T_{s}$ and $B \triangleq F T_{s}$ are the discretized matrices, obtained from the backward Euler approximation $[13,14]$ for short sampling intervals of up to several tens of $\mu \mathrm{s}$. Note that the term $I$ is the identity matrix of appropriate dimension.

Considering the grid terminal voltage $V_{g}$ as sinusoidal and balanced, one can estimate the future value of output current 
and use it to predict the future value of controlled variables as the following

$$
\begin{aligned}
i_{o}^{\alpha \beta}(k+1) & =\frac{T_{s}}{L}\left[V_{m}{ }^{\alpha \beta}(k)-V_{g}^{\alpha \beta}(k)-R i_{o}^{\alpha \beta}(k)\right] \\
& +i_{o}^{\alpha \beta}(k)
\end{aligned}
$$

\section{Model Predictive Power Control of the Case Study}

The direct power control (DPC) pursues to control a power converter by employing the active and reactive powers as the control variables. DPC has become a popular control strategy due to its simplicity, outstanding transient performance, and robustness. Although DPC directly selects the power switch states to follow the desired active and reactive powers, the resulting switching frequency is varying [15].

The block diagram of the MPC based DPC for a $2 \mathrm{~L}-3 \mathrm{Ph}$ inverter is depicted in Fig. 5. The discrete-time mathematical model of the system is used to compute the predictions of the output until time $k+N$ for a given sequence of inverter voltage vectors and measurements at time $k$. The optimal sequence of voltage vectors that minimize the objective function is selected and the first element of the resultant control sequence is applied based on the receding horizon control principle. This algorithm is repeated at each sampling time.

\section{A. One-Step Prediction}

The objective of the MPC controller is to permit flexible power regulation, in order to improve the power quality. In the stationary reference $\alpha \beta$ frame and for a balanced three-phase system, the instantaneous active and reactive powers can be defined as

$$
\begin{array}{r}
P(k+1)=\frac{3}{2}\left[\begin{array}{r}
V_{g \alpha}(k+1) \cdot i_{o \alpha}(k+1) \\
\left.+V_{g \beta}(k+1) \cdot i_{o \beta}(k+1)\right]
\end{array}\right. \\
Q(k+1)=\frac{3}{2}\left[\begin{array}{r}
V_{g \alpha}(k+1) \cdot i_{o \beta}(k+1) \\
\left.-V_{g \beta}(k+1) \cdot i_{o \alpha}(k+1)\right]
\end{array}\right.
\end{array}
$$

The current and grid voltage are measured at the same instant and applied as the input for the predictive model that calculates the values of $P(k+1)$ and $Q(k+1)$ at the next sampling time for each of the possible switching states of the inverter. The objective function is defined as

$$
\begin{aligned}
J=\left[P^{*}(k+1)-\right. & P(k+1)]^{2} \\
+ & {\left[Q^{*}(k+1)-Q(k+1)\right]^{2} }
\end{aligned}
$$

The MPC chooses the finest voltage-vector and corresponding switching positions by minimizing the objective function and the switching positions are obtained through an exhaustive search; for example in this case, there will be seven voltage vector positions as the redundant vector can be neglected.

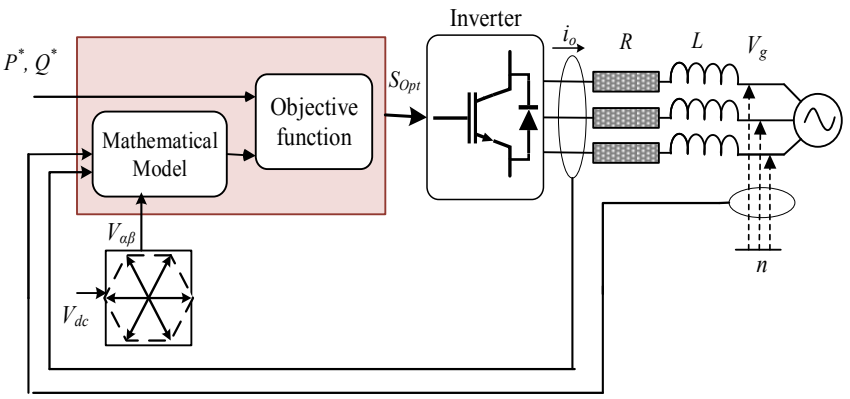

Fig. 5. Block diagram of the grid-connected inverter with MPC controller

\section{B. A Longer Prediction Horizon}

The control delay caused by the digital data processor is a crucial factor that needs to be reflected in the real-time implementation of MPC schemes. The calculation time is considerable, and the delay between the measurements and actuation can impose some problems if not addressed. Furthermore, in distributed power generations, the lower the switching frequency is, the less the power loss will be.

A key solution to compensate this time delay is to take into account the computation time and apply the selected switching state after the next sampling instant. In order to tackle these problems, a longer prediction horizon is required. Therefore, the continuous time state space of the system can be improved by

$$
\frac{d x(t)}{d t}=D x(t)+F u(t-\tau)
$$

where $\tau$ is the required time delay for controller to minimize the objective function of the algorithm. If we assume that $\tau$ is equal to the sampling time and is long enough for exhaustive search of the optimal switching positions, a two-step prediction can be implemented.

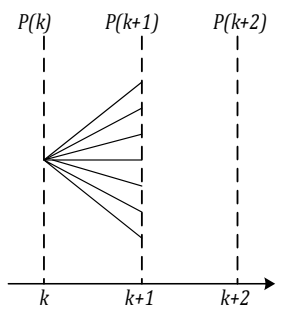

a)

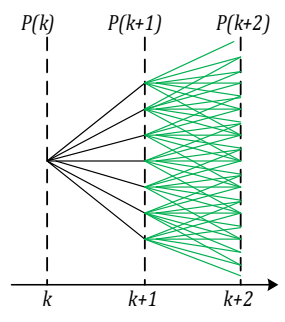

b)

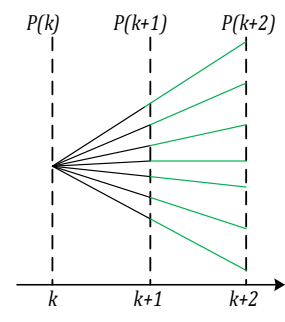

c)
Fig. 6. Prediction of the output voltage vectors considering different input sequences. a) One-step prediction. b) Two-step prediction considering all possibilities. c) Simplified two-step prediction

With the one-step prediction, only seven voltage vectors are considered. When two steps are considered for prediction, the total number of the voltage vectors will be $m^{N}$, where $m$ is the number of possible voltage vectors and $N$ the prediction horizon. In this case, the number of possible trajectories for the voltage vector is 49 . In fact, as the prediction horizon is enlarged, the number of decision variables is increased. Since the computational complexity grows, the experimental implementation of the algorithm will be difficult. To simplify this approach, it is possible to eliminate the redundant voltage 
vectors in order to reduce the number of calculations. Here, instead of 49 possible trajectories for the voltage vector, 7 voltage vectors are assumed as the simplified two-step prediction. Different approaches discussed in this work are illustrated in Fig. 6 for one- and two-step prediction horizons.

The compensated control algorithm is shown in Fig. 7. In steady state, to reduce the switching frequency, the controller needs to minimize possible state alterations. As such, $S_{E r r}$, characterizing the error between the neighboring switching states, has been considered as the second term in the revised cost function as

$$
\begin{gathered}
J=\sum E_{P w r}(k+2)+\lambda S_{E r r} \\
E_{P w r}=\left[P^{*}(k+2)-P(k+2)\right]^{2} \\
+\left[Q^{*}(k+2)-Q(k+2)\right]^{2} \\
S_{E r r}=\sum_{h=a, b, c}\left(S_{h}(k+2)-S_{h}(k+1)\right)^{2}
\end{gathered}
$$

where $S_{h}(k+1)$ and $S_{h}(k+2)$ are the switching state of the phase $h=\{a, b, c\}$ in the current sampling interval and the next sampling interval, respectively, $\lambda$ is the weighting coefficient (between 0 and 1) with a large value showing a higher priority for the second term. $S_{h}$ can be either 0 or 1 , where $S_{h}=0$ means that the upper switch is off and the lower one is on, and $S_{h}=1$ means the opposite. For example, supposing that $V_{2}(110)$ and $V_{3}(010)$ are applied at the current interval and next interval correspondingly, then the second term of the objective function can be determined via (19b) as $S_{E r r}=(1-0)^{2}+(1-1)^{2}+(0-0)^{2}=1$.

\section{NUMERICAL SimULATION}

\section{A. Simulation Results}

Simulation of a $2 \mathrm{~L}-3 \mathrm{Ph}$ gird-connected inverter with $\mathrm{RL}$ filters and loads is executed by using MATLAB/Simulink. The model is shown in Fig. 3, with the system parameters listed in Table I. Fig. 8 (a) and (b) demonstrate the controller performances with one- and two-step predictions, respectively. Initially the active and reactive powers are set to zero. While the active power reference is decreased from 0 to $-3 \mathrm{~kW}$ at $0.02 \mathrm{~s}$ and is back to $0 \mathrm{~W}$ at $0.04 \mathrm{~s}$. After that, the active power reference is increased to $1 \mathrm{~kW}$ at $0.06 \mathrm{~s}$ and then is kept at 1 $\mathrm{kW}$, whereas the reactive power reference is changed to $-2,0$, and $1 \mathrm{kVAr}$ at $0.04 \mathrm{~s}, 0.05 \mathrm{~s}$ and $0.06 \mathrm{~s}$, respectively.

It can be seen that the by applying the delay compensation scheme, the power ripples have been reduced significantly. Unity power factor can be achieved by both methods but MPC with two-step prediction is able to track the reference more accurately, with less power ripples. As can be seen in Fig.9, the load current and voltage are stable without any deterioration, and the DG can meet the demand in cooperation with the utility grid.

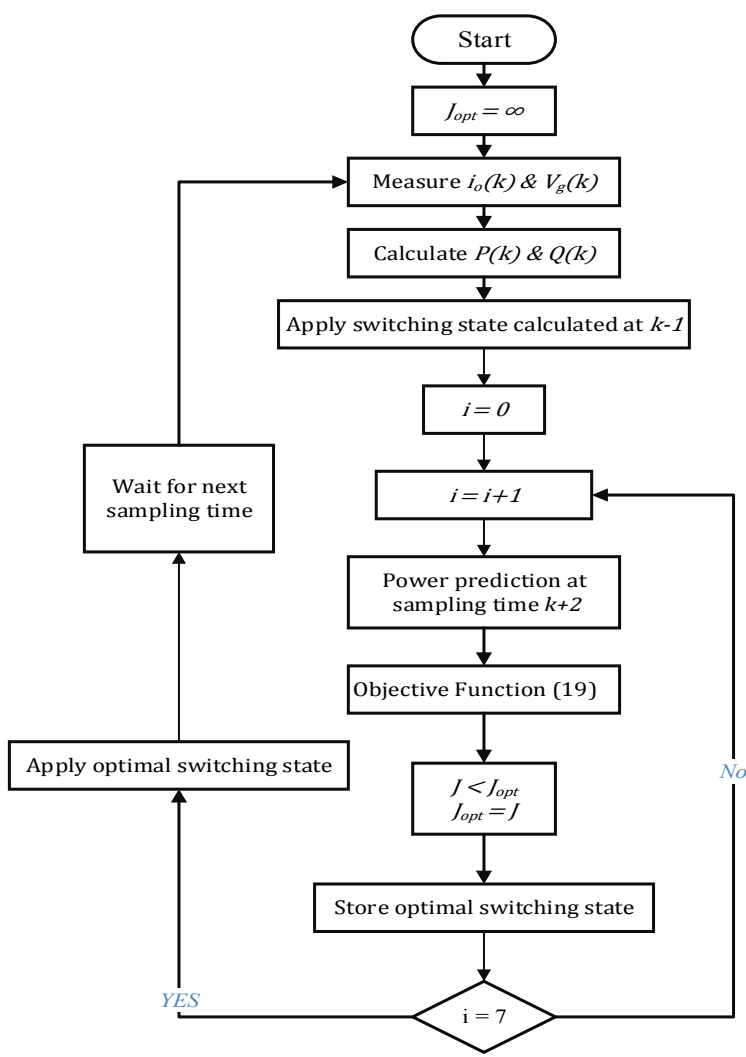

Fig. 7. Model predictive control algorithm flow diagram

TABLE I. System Parameters

\begin{tabular}{lcc}
\hline Filter resistance & $R$ & $1 \Omega$ \\
Filter inductance & $L$ & $7.8 \mathrm{mH}$ \\
Grid voltage & $V_{g}$ & $120 \mathrm{~V}$ \\
Dc source voltage & $V_{d c}$ & $400 \mathrm{~V}$ \\
Voltage frequency & $f$ & $50 \mathrm{~Hz}$ \\
Sampling period & $T_{S}$ & $25 \mu \mathrm{s}$ \\
\hline
\end{tabular}

\section{B. Quantitative Comparison Study}

A quantitative comparison of MPC methods in simulation is carried out. In order to get a better comparison of the control schemes, quantitative keys of the average switching frequency, and power ripples are tabulated in Table II. The measurements are provided through the MATLAB.

\begin{tabular}{ccccc}
\multicolumn{5}{c}{ TABLE II. Quantitative Comparison } \\
\hline MPC with & $\boldsymbol{f}_{\boldsymbol{s}}(\boldsymbol{H z})$ & $\boldsymbol{f}_{\boldsymbol{s w}}(\boldsymbol{H z})$ & $\boldsymbol{P}_{\text {rip }}(\boldsymbol{W})$ & $\boldsymbol{Q}_{\text {rip }}($ Var $)$ \\
One-step & $40 \mathrm{k}$ & 9551 & 350 & 450 \\
$\begin{array}{c}\text { Two-step } \\
\boldsymbol{\lambda}=\mathbf{0 . 7}\end{array}$ & $40 \mathrm{k}$ & 5267 & 84.38 & 96.54 \\
$\begin{array}{c}\text { Two-step } \\
\boldsymbol{\lambda}=\mathbf{0 . 2}\end{array}$ & $40 \mathrm{k}$ & 5666 & 98.49 & 127.93 \\
\hline
\end{tabular}

It can be seen that the performance of the MPC with twostep prediction is much better than that of one-step prediction. After using the longer horizon to compensate delay, the power ripples are reduced noticeably. With the switching frequency reduction method, the two-step prediction with $\lambda=0.7$ reduces the switching frequency to less than half of the value in the one-step prediction. 

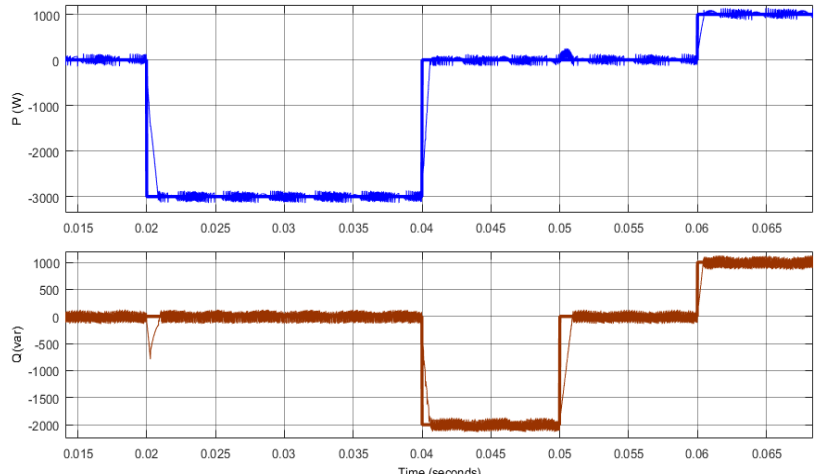

a)
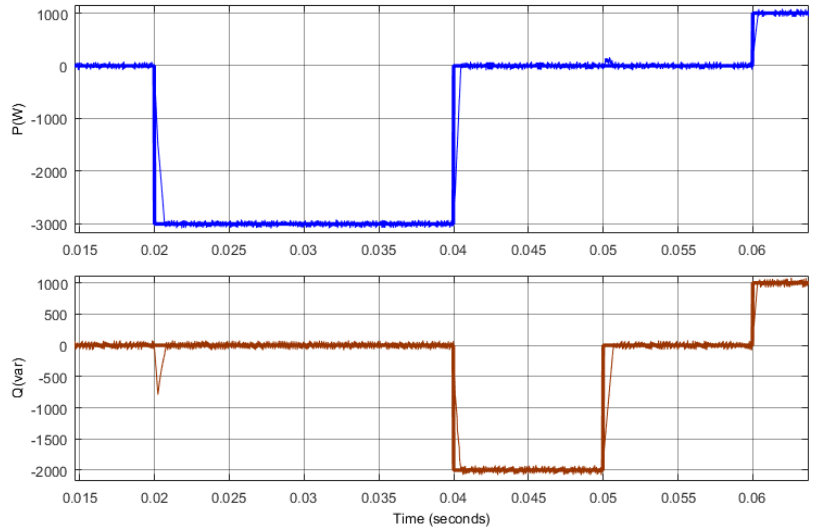

b)

Fig. 8. Numerical simulation results of model predictive control a) Onestep prediction horizon b) A longer prediction horizon $\left(\tau=T_{S}\right)$

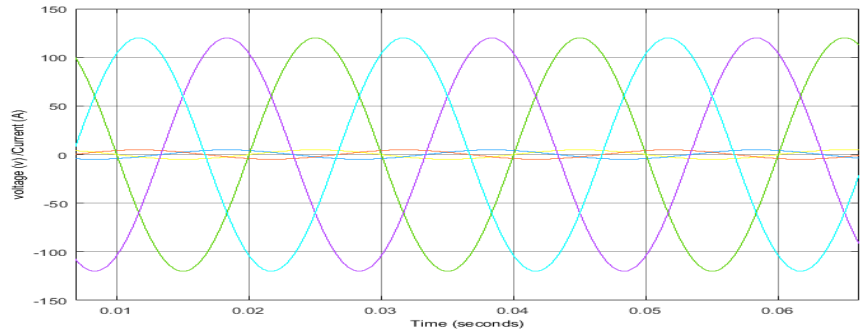

Fig. 9. The voltage and current of the Load

\section{CONCLUSION AND FUTURE WORK}

In this study, two emerging predictive control procedures, namely, one-step predictive control with reference tracking and MPC with longer prediction horizons are reviewed and compared. Both schemes are used for direct power controllers. MPC takes the benefit of the discrete nature of power converters and advancement of microprocessors in order to reduce the amount of calculations.

An MPC based finite control set technique is applied to a power inverter with the DPC strategy. It can reduce the huge amount of calculations in the online implementation of MPC. The proposed method leads to good performance of the power tracking ability in both steady and transient states, which can be used as a general control approach for distributed generation units to achieve grid-tied operation. By changing the cost function properly, different control objectives can be achieved. The longer prediction horizon can reduce the power ripples considerably. For the future work, improving power quality and system stability through multi-objective cost function will be investigated. Furthermore, the study will focus on the optimal length of the prediction horizon.

\section{REFERENCES}

[1] J. M. Carrasco, L. G. Franquelo, J. T. Bialasiewicz, E. Galván, R. C. P. Guisado, M. Á. M. Prats, et al., "Power-electronic systems for the grid integration of renewable energy sources: A survey," Industrial Electronics, IEEE Transactions on, vol. 53, pp. 10021016, 2006.

[2] S. Vazquez, A. Marquez, R. Aguilera, D. Quevedo, J. I. Leon, and L. G. Franquelo, "Predictive optimal switching sequence direct power control for grid-connected power converters," Industrial Electronics, IEEE Transactions on, vol. 62, pp. 2010-2020, 2015.

C. Bhattacharjee and B. K. Roy, "Advanced fuzzy power extraction control of wind energy conversion system for power quality improvement in a grid tied hybrid generation system," IET Generation, Transmission \& Distribution, vol. 10, pp. 1179-1189, 2016.

[4] R. Guzman, L. G. de Vicuna, J. Morales, M. Castilla, J. Miret, and J. Torres-Martinez, "Sliding-mode control for a three-phase shunt active power filter in natural frame," in Industrial Electronics Society, IECON 2015-41st Annual Conference of the IEEE, 2015, pp. 001211-001216.

[5] J. J. More, P. F. Puleston, C. Kunusch, and M. A. Fantova, "Development and implementation of a supervisor strategy and sliding mode control setup for fuel-cell-based hybrid generation systems," Energy Conversion, IEEE Transactions on, vol. 30, pp. 218-225, 2015.

[6] J. Rodriguez, M. P. Kazmierkowski, J. R. Espinoza, P. Zanchetta, H. Abu-Rub, H. A. Young, et al., "State of the art of finite control set model predictive control in power electronics," Industrial Informatics, IEEE Transactions on, vol. 9, pp. 1003-1016, 2013.

M. Rivera, V. Yaramasu, A. Llor, J. Rodriguez, B. Wu, and M. Fadel, "Digital predictive current control of a three-phase four-leg inverter," Industrial Electronics, IEEE Transactions on, vol. 60, pp. 4903-4912, 2013.

V. Yaramasu, B. Wu, and J. Chen, "Model-predictive control of grid-tied four-level diode-clamped inverters for high-power wind energy conversion systems," Power Electronics, IEEE Transactions on, vol. 29, pp. 2861-2873, 2014.

D. Quevedo and T. Geyer, "Model predictive control with reference tracking," ed: Google Patents, 2015.

T. Geyer and D. E. Quevedo, "Performance of multistep finite control set model predictive control for power electronics," Power Electronics, IEEE Transactions on, vol. 30, pp. 1633-1644, 2015.

M. Mahdavi Aghdam, L. Li, J. Zhu, and O. Palizban, "Finite Control Set Model Predictive Control-A Powerful Control Algorithm for Grid-Connected Power Converters," in Industrial Electronics and Applications (ICIEA), 2016 11th IEEE Conference on, Hefei, China, 2016.

J. Hu, J. Zhu, G. Lei, G. Platt, and D. G. Dorrell, "Multi-objective model-predictive control for high-power converters," Energy Conversion, IEEE Transactions on, vol. 28, pp. 652-663, 2013.

S. Kouro, P. Cortés, R. Vargas, U. Ammann, and J. Rodríguez, "Model predictive control-A simple and powerful method to control power converters," Industrial Electronics, IEEE Transactions on, vol. 56, pp. 1826-1838, 2009.

V. Yaramasu, M. Rivera, M. Narimani, B. Wu, and J. Rodriguez, "Finite State Model-based Predictive Current Control with Twostep Horizon for Four-leg NPC Converters," Journal of Power Electronics, vol. 14, pp. 1178-1188, 2014.

[15] J. Hu, J. Zhu, and D. G. Dorrell, "In-depth study of direct power control strategies for power converters," Power Electronics, IET, vol. 7, pp. 1810-1820, 2014. 